\title{
Nonlinear boundary oscillations in strongly correlated electron quantum wires
}

\author{
Holger Frahm \\ Institut für Theoretische Physik, Universität Hannover, D-30167 Hannover, Germany \\ Andrei A. Zvyagin \\ Institut für Theoretische Physik, Universität Hannover, D-30167 Hannover, Germany \\ and B. I. Verkin Institute for Low Temperature Physics and Engineering of the National Ukrainian Academy of Sciences, \\ 310164 Kharkov, Ukraine \\ (Received 20 September 1996)
}

\begin{abstract}
We study the influence of a boundary point contact voltage on Coulomb blockadelike oscillations of the conductance in mesoscopic quantum wires of strongly correlated electrons. Bethe ansatz techniques allow one to understand lattice boundary effects together with nonconformal many-body behavior for such systems. We predict a nonlinear dependence of the initial (coherent) shift on oscillations with boundary potentials. The results are obtained for both spinless and spin cases. [S0163-1829(97)05503-3]
\end{abstract}

Both theoretical and experimental interest in properties of systems with a boundary (backscattering impurity) has risen recently. On the theoretical side this is connected with the development of exact methods to deal with open boundary conditions. $^{1-3}$ Important progress has been achieved using bosonization (both Abelian and non-Abelian) and conformal field theory descriptions of Luttinger liquids with boundary (or impurity) field. ${ }^{4}$ However, this approach is usually limited to small coupling constants (less than the effective Fermi velocities for the corresponding noninteracting system) and uses a linearized dispersion for the elementary excitations which neglects effects of the finite lattice spacing. A proper treatment of these may lead to answers differing drastically from the linearized situation. These difficulties do not arise for exact solutions of the lattice system obtained using the Bethe ansatz method. ${ }^{5}$ Combining Bethe ansatz calculations with results from boundary conformal field theory, very recently several important results have been obtained for a number of models, e.g., the $S=1 / 2 X X Z$ spin chain, ${ }^{3}$ the quantum sine-Gordon (or Thirring field theory) model, ${ }^{2}$ and the Hubbard model with boundary fields. ${ }^{6}$ All of these solutions manifest the strong difference between the behavior of bulk and surface energies, or long-range asymptotics for low-lying (gapless) boundary and bulk excitations. On the other hand, recent experiments on quantum wires with impurities or boundaries manifest interesting features, e.g., Coulomb blockadelike behavior, proximity effects for quantum wires connected through leads (contacts) to superconductors, and quantum topological effects such as the Aharonov-Bohm effect for ring geometries. 7,8

In this report we propose to study new nonlinear effects due to boundaries or backscattering impurities in chiral conductors. Since we deal with exactly solvable models, our results apply to the strong-coupling regime and all lattice effects are taken into account. The transfer of (virtual) elementary excitations from one boundary to the other alters dramatically mesoscopic properties of the system. We point out that the phase shift due to this transfer provides additional information about the nature of electron interaction in quantum wires.
To start let us consider a model of spinless interacting electrons on a linear lattice of $L$ sites related to the $X X Z$ model by means of the Jordan-Wigner transformation

$$
\begin{aligned}
\mathcal{H}= & \sum_{j=1}^{L-1}\left[-\frac{1}{2}\left(a_{j}^{\dagger} a_{j+1}+\text { H.c. }\right)+\Delta\left(n_{j}-\frac{1}{2}\right)\left(n_{j+1}-\frac{1}{2}\right)\right] \\
& -\mu \sum_{j=1}^{L} n_{j}-\left(\mu_{+} n_{1}+\mu_{-} n_{L}\right),
\end{aligned}
$$

where $a_{j}\left(a_{j}^{\dagger}\right)$ are annihilation (creation) operators for electrons in site $j$, and $n_{j} \equiv a_{j}^{\dagger} a_{j}$ is the number of electrons at site $j$, and $\Delta \geqslant 0$ is the strength of the (repulsive) interaction. $\mu$ and $\mu_{ \pm}$are chemical potentials for bulk electrons and boundary potentials at the right and left boundary, respectively. In an experiment this situation could be realized by applying boundary external voltages (for chiral electrons with one scatterer as a plunger voltage applied to this point, one edge would be free, i.e., $\mu_{+}=0$ ). A different realization of boundary fields could be potentials due to proximity effect of different bulk samples (leads), producing point contact potential difference.

The mesoscopic effects we want to study in this report reveal themselves for system sizes less than the phase coherence length $L_{\mathrm{ph}}$. Hence we consider the conducting regime of a repulsive interaction with gapless excitations, $\Delta=\cos \gamma \leqslant 1$, in the insulating case $(\Delta>1)$ they are suppressed exponentially. Using the Yang-Baxter and reflection equations we diagonalize the Hamiltonian (1). The eigenstates in the $N$ particle sector are characterized by a set of $N$ rapidities $u_{j}$, which are determined by the Bethe ansatz equations ${ }^{9}$

$$
\begin{gathered}
2 L \phi\left(u_{j}, \gamma / 2\right)+\sum_{x= \pm} \phi\left(u_{j}, \Gamma_{x}\right)= \\
2 \pi I_{j}+\sum_{l=1, l \neq j}^{N} \phi\left(u_{j}-u_{l}, \gamma\right) \\
+\phi\left(u_{j}+u_{l}, \gamma\right) .
\end{gathered}
$$

Here $\phi(u, \gamma)=2 \arctan [\cot \gamma \tanh u], \quad \Gamma_{ \pm}=\left(2 \mu_{ \pm}-\Delta+e^{i \gamma}\right) /$ $\left[\left(2 \mu_{ \pm}-\Delta\right) e^{i \gamma}+1\right]$, and $I_{j}$ are positive integers. The corre- 
sponding eigenvalue of the Hamiltonian (1) is

$$
E=(L-1) \frac{\Delta}{4}-\sum_{j=1}^{N}\left[\mu+2 \sin \gamma \phi^{\prime}\left(u_{j}, \gamma\right)\right] .
$$

Expanding the ground-state energy in powers of $L$ one obtains $E=L E_{\infty}+E_{\text {surf }}+\left(L^{-1}\right) E_{\text {mes }}+\cdots$. Here $E_{\infty}$ is the energy density of the infinite system, $E_{\text {surf }}$ is the surface energy connected with the boundaries (or impurity), and $E_{\text {mes }}$ is the mesoscopic energy, which - as in systems with periodic boundary conditions (see, e.g., Refs. 10 and 11)_determines the asymptotic (long-distance) behavior of correlation functions via conformal invariance ${ }^{12}$ and topological quantum effects in one-dimensional (1D) systems. ${ }^{13}$ As discussed above, for $\Delta>1$ the elementary excitations have a gap, leading to finite-size corrections to the energy $\propto \exp \left(-L E_{\text {gap }} / \hbar v^{F}\right)$. In the following we concentrate on the mesoscopic energy that determines the mesoscopic Coulomb blockadelike oscillations in conducting quantum wires without (superconducting) ordering. ${ }^{14}$

Following Ref. 15 one obtains the finite-size (mesoscopic) energy with open boundaries: for large $L$ the sums in Eqs. (2) and (3) can be replaced by integrals using the EulerMaclaurin formula giving

$$
E_{\mathrm{mes}}=\pi v^{F}\left[\frac{\left[\Delta N-\Theta\left(\mu_{ \pm}\right)\right]^{2}}{2 \xi^{2}(Q)}+N^{+}-\frac{1}{24}\right],
$$

where $v^{F}$ plays the role of a Fermi velocity and is determined by the dispersion relation near the Fermi point, see Ref. 6, $\Delta N=N-n(\mu) L$ with $n$ being the band filling, and $N^{+}>0$ is the number of (bosonic) particle-hole excitations near the Fermi point. The dressed charge $\xi(Q)$ is a measure for the interactions determining the critical properties of the system. ${ }^{11}$ It can be interpreted as the number of electrons for each of the elementary excitations forming the Fermi sea of holons. The boundary potentials enter Eq. (4) through the "boundary shift" $\Theta\left(\mu_{ \pm}\right)$, which is the corresponding contribution to the integrated density $\rho$ of the $u_{j}$, namely, $(1 / 2) \int_{-Q}^{Q} \rho(u) d u=n(\mu)-\Theta\left(\mu_{ \pm}\right) / L$.

To calculate the dependencies of Fermi velocity, dressed charge, and the boundary shift $\Theta$ on coupling parameter $\gamma$, and bulk $(\mu)$ and boundary $\left(\mu_{ \pm}\right)$chemical potentials we have to solve linear integral equations of the structure $y(u)=y^{0}(u)-(1 / 2 \pi) \int_{-Q}^{Q} \phi^{\prime}(u-v) y(v) d v$ (for the open boundary case, only symmetric limits of integration are possible). For the dressed charge $y(u)=\xi(u)$ the driving term is just $y^{0}(u)=1$. At half filling corresponding to $Q=\infty$ these integral equations can be solved by Fourier transformation resulting in $\xi(Q=\infty)=\sqrt{\pi / 2(\pi-\gamma)}$ for the dressed charge and

$$
2(\pi-\gamma) \Theta\left(\mu_{ \pm}\right)=\gamma-\sum_{x= \pm} \arctan \left(\cot \frac{\gamma 2 \mu_{x}-\Delta+1}{22 \mu_{x}-\Delta-1}\right)
$$

Equation (5) shows the strongly nonlinear dependence of $\Theta$ on the boundary potentials: increasing $\mu_{ \pm}$from negative values the boundary shift increases monotonically until $2 \mu_{ \pm}=1+\Delta$ where $\Theta$ jumps by $-\pi / 2(\pi-\gamma)$. Beyond this point, $\Theta$ continues to increase monotonically, reaching

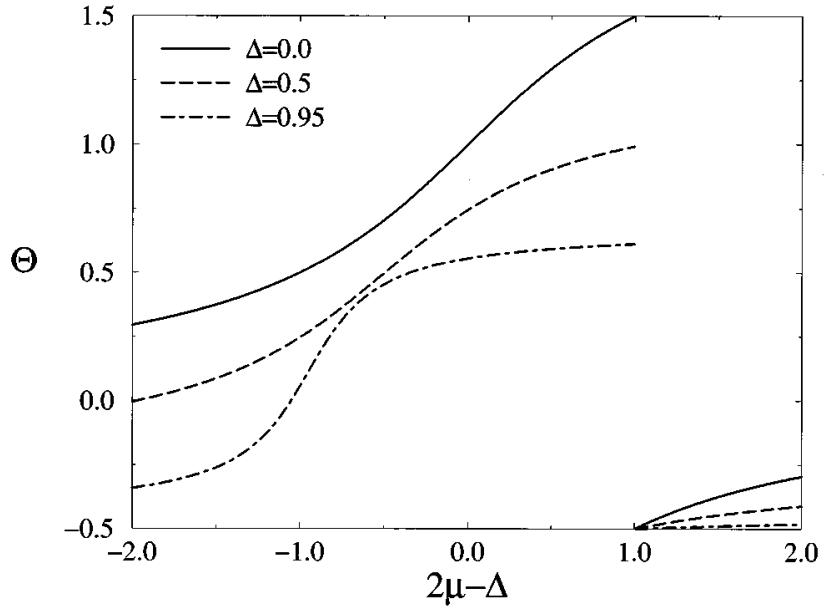

FIG. 1. $\Theta\left(\mu_{+}=\mu_{-}\right)$vs boundary chemical potential for the spinless fermion system (1) at half filling $n=\frac{1}{2}$.

$-(\pi-2 \gamma) / 2(\pi-\gamma)$ at $\mu_{ \pm} \rightarrow \infty$, see Fig. 1. Since the effect of the two boundaries enters additively in Eq. (5) it is possible to cancel this effect completely by suitable tuning of $\mu_{ \pm}$. The discontinuity at the points $2 \mu_{ \pm}=1+\Delta$ indicates the creation of surface bound states, which also changes the surface energy $E_{\text {surf }}$. To conserve the zero topological charge of the ground state at half filling these edge bound states have to be created either in pairs or together with a bulk excitation (kink). This creation of a bulk excitation is the microscopic origin of the oscillations that we will study below.

The mesoscopic energy in the ground state of the system is obtained by minimization of Eq. (4) with respect to $\Delta N$, which is achieved by replacing $N-n(\mu) L-\Theta\left(\mu_{ \pm}\right)$with its fractional part to the nearest integer. ${ }^{16}$ This leads to periodic oscillations of the mesoscopic energy connected to the change of the number of electrons in the system. These oscillations can be observed when the external bulk potential (an external voltage) is varied, and are just the well-known Coulomb blockadelike oscillations. They are a consequence of the finite lattices considered here: although they are determined from finite-size data such as $E_{\text {mes }}$ the conformal properties are characteristics of a continuum model. Since only a discrete set of densities can be realized on a finite lattice one finds a nonanalytic dependence of the mesoscopic energy on the chemical potential. ${ }^{16}$ Variation of boundary (point contact) potentials allows for the investigation of the nonlinear (coherent) shift of the Coulomb blockadelike oscillations due to these potentials. Similar coherent oscillation shifts were observed very recently in experiments for the loop geometry with a quantum dot embedded into a metallic ring. ${ }^{8}$ Here both Coulomb blockade and Aharonov-Bohm oscillations of persistent currents were measured, the latter existing due to ring geometry (see Ref. 17). In the noninteracting system, $\Delta=0$, the discontinuity of $\Theta$ is at $2 \mu_{ \pm}=1$ where one of the bulk electrons is transferred into the boundary bound state. In this case only the monotonic shift of the initial phase for Coulomb blockadelike oscillations can be observed. For $\Delta=1$, the largest value giving a conducting ground state, $\Theta$ degenerates to a step function taking values 0 and $\pm 1 / 2$. Away from half filling the Coulomb blockadelike oscillations persist for bulk voltages less than the critical one, 


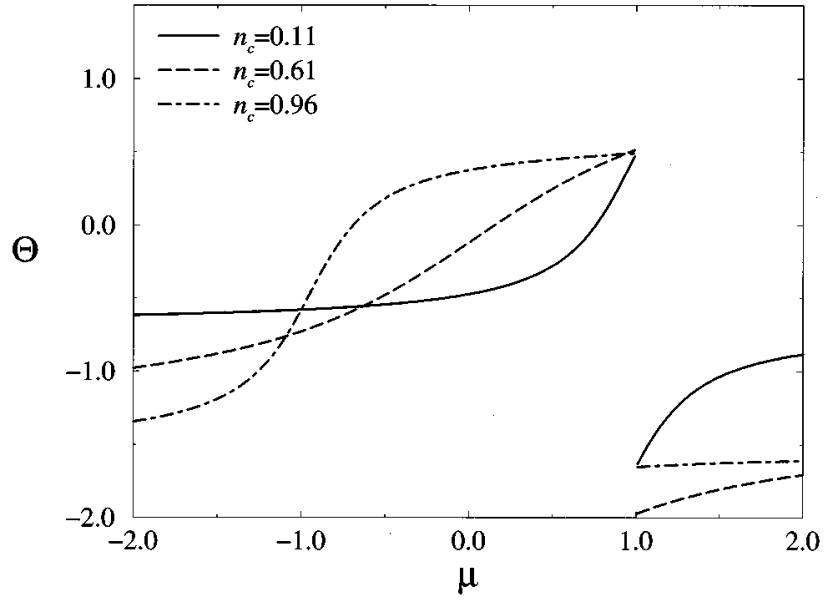

FIG. 2. $\Theta\left(\mu_{+}=\mu_{-}\right)$vs boundary chemical potential for several values of the density $n_{c}$ in the Hubbard model with $U=1$.

which corresponds to the gap opening for excitation (complete band filling), and $\Theta$ reaches zero at such critical point. Generally, one finds that the discontinuity of $\Theta$ decreases with the interaction as seen in Fig. 1.

For the case of spin fermions we consider the repulsive Hubbard model with the Hamiltonian

$$
\begin{aligned}
\mathcal{H}= & \sum_{j=1}^{L-1}\left[-\sum_{\sigma}\left(a_{j, \sigma}^{\dagger} a_{j+1, \sigma}+\text { H.c. }\right)+4 U n_{j \uparrow} n_{j \downarrow}\right] \\
& -\mu \sum_{j=1}^{L} \sum_{\sigma} n_{j \sigma}-\sum_{\sigma}\left(\mu_{+} n_{1, \sigma}+\mu_{-} n_{L, \sigma}\right),
\end{aligned}
$$

where $U \geqslant 0$ is the Hubbard constant and $\sigma=\uparrow, \downarrow$ is the spin of the electrons. Here the states are characterized by two sets of quantum numbers, namely, $N$ quasimomenta $k_{j}$ (connected with charged holon excitations) and $M$ rapidities, $u_{j}$, connected with spinons ( $N$ and $M$ are numbers of electrons and down spin electrons, respectively). Constructing Bethe ansatz equations, and calculating the finite-size corrections for open boundary conditions as above we obtain for the relevant terms in the mesoscopic energy at fillings $n<1$ :

$$
\begin{aligned}
E_{\mathrm{mes}}= & \pi v_{c}^{F}\left[\frac{\left[\left(\Delta N-\Theta_{c}\right) Z_{22}-\left(\Delta M-\Theta_{s}\right) Z_{21}\right]^{2}}{2(\operatorname{det} Z)^{2}}\right] \\
& +\pi v_{s}^{F}\left[\frac{\left[\left(\Delta N-\Theta_{c}\right) Z_{12}-\left(\Delta M-\Theta_{s}\right) Z_{11}\right]^{2}}{2(\operatorname{det} Z)^{2}}\right] .
\end{aligned}
$$

Here the Fermi velocities for spin and charge excitations are different in general, $\Delta N=N-n L, \Delta M=M-m L, n$ and $m$ being band filling and density of spin $\downarrow$ electrons, respectively. Without external magnetic fields one has $m=n / 2$ and the components of the dressed charge matrix can be parametrized by a single number $\xi$ as $Z_{11}=2 Z_{12}=\xi, Z_{22}=2^{-1 / 2}$, $Z_{21}=0 .{ }^{10}$ The boundary shifts $\Theta_{c}=2 \Theta_{s} \equiv \Theta\left(\mu_{ \pm}\right)$are functions of the boundary chemical potentials. Using this we obtain the following expression for the mesoscopic energy:

$$
E_{\mathrm{mes}}=\pi v_{c}^{F} \frac{(\Delta N-\Theta)^{2}}{2 \xi^{2}}+\pi v_{s}^{F} \frac{(\Delta N-2 \Delta M)^{2}}{4} .
$$

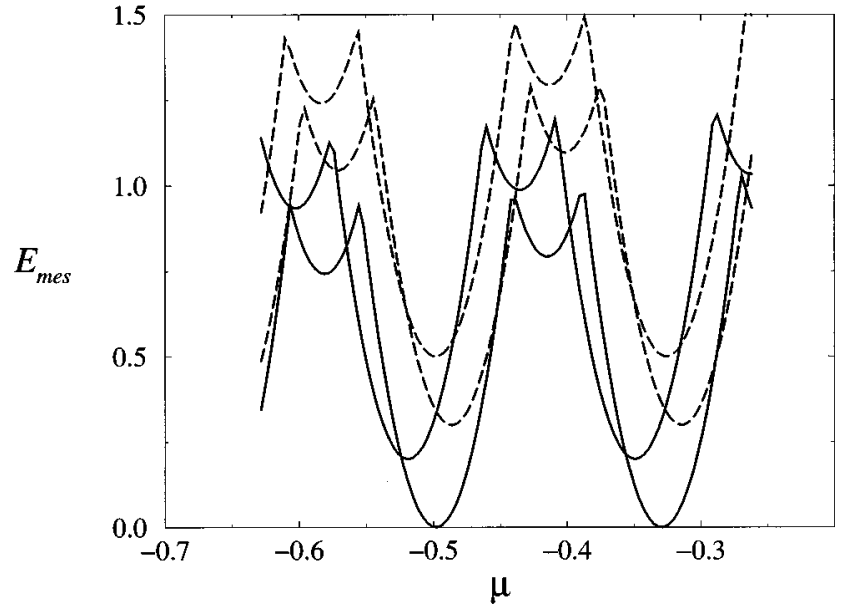

FIG. 3. Oscillations of mesoscopic energy (7) vs chemical potential for a Hubbard model $(U=1)$ on a lattice of $L=50$ sites for boundary potentials $\mu_{+}=\mu_{-}=0.5,0.9,1.1,1.5$ (bottom to top).

Here $\Theta=(1 / 2)\left[\int_{-Q}^{Q} \theta(k) d k-1\right]$, where $\theta(k)$ is determined from the integral equation given above with kernel $\phi^{\prime}\left(k, k^{\prime}\right)=\cos k \int_{-\infty}^{\infty} d \omega \exp \left[-U|\omega|+i\left(\sin k-\sin k^{\prime}\right) \omega\right] /$ $2 \cosh (U \omega)$, and driving term

$$
\theta^{0}(k)=\frac{1}{2 \pi} \sum_{x= \pm} \frac{1-\mu_{x}^{2}}{1+\mu_{x}^{2}-2 \mu_{x} \cos k}-\frac{\cos k}{4 U \cosh [(\pi / 2 U) \sin k]} .
$$

For the dressed charge $\xi(k)$, the equation has similar structure as for $\theta(k)$ with the driving term being unity and with the kernel replaced by its transpose. $Q$ now defines the Fermi point for quasimomenta of charge excitations, and depends on bulk chemical potential. In general, Fermi velocities, dressed charge matrix and boundary shifts depend on external magnetic fields (both bulk and boundaries) also, but this case will be reported elsewhere. ${ }^{18}$

Naturally, the situation is similar to the case of repulsive spinless electrons studied above. The boundary shift now depends both on the value of Hubbard constant and external potential (bulk voltage). The dependence of boundary shift $\Theta$ on $\mu_{ \pm}$is shown in Fig. 2. Again, $\Theta$ jumps at boundary potentials $\mu_{ \pm}=1$ by an amount depending on the interaction [note that the energy scales in Eqs. (1) and (6) differ by a factor of 2]. In the noninteracting case, $U=0$, we simply have two copies of the spinless electrons considered above: the boundary shift depends monotonically on $\mu_{ \pm}$and jumps by -2 when surface bound states are created. The strongcoupling limit, $U \rightarrow \infty$, corresponds to the free spinless electron case, because Hubbard repulsion works as an additional Pauli principle. In this limit, the boundary shift is found to be $\Theta\left(\mu_{ \pm}\right)=(1 / \pi) \Sigma_{x= \pm} \arctan \left\{\left[\left(1+\mu_{x}\right) /\left(1-\mu_{x}\right)\right] \tan Q / 2\right\}-\frac{1}{2}$, where $Q=\pi n$. Note that the jump of $\Theta$ at boundary potentials $\mu_{ \pm}=1$ is -1 for any (metallic) band filling; see Fig. 2 . Going to large but finite values of the Hubbard constant this jump increases to $\Delta \Theta=-1-(\ln 2 / U) \sin \pi n$. For intermediate values of $U$ the equations have to be solved numerically. Again one finds oscillations of $E_{\text {mes }}$ with the particle number or, equivalently, the bulk chemical potential with a jump in the phase at $\mu_{ \pm}=1$ (see Fig. 3). The size of this jump changes from -2 in the noninteracting case to -1 in the 
strong-coupling limit, depending on $U$ and the filling in between. The main effect of the massless spinon excitations on the oscillations is a parity effect leading to a period doubling: for an odd number of electrons, the ground state contains a virtual spinon shifting $E_{\text {mes }}$ by $\pi v_{s}^{F} / 4$. Since $v_{s}^{F} \rightarrow 0$ for $U \rightarrow \infty$ this effect disappears in the strong coupling limit.

The oscillations studied in this report can be observed for sufficiently small temperatures, $T \sim \hbar v^{F} / L$. For higher temperatures mesoscopic oscillations decrease exponentially with temperature and the shape of sharp resonances is smeared (sawtoothlike oscillations into cosinelike ones).

To conclude, we have studied the effect of boundary point contact potentials on the coherent mesoscopic oscillations in strongly correlated quantum wires. We predict a nonlinear behavior for (coherent) initial shift(s) of mesoscopic Coulomb blockadelike oscillations due to boundary shift $\Theta$. A measurement of the discontinuity of this phase shift near the boundary potential $\mu_{ \pm}=1$ allows for a direct determination of the effective interaction constants for 1D strongly correlated electron systems. Our results were obtained both for spinless and for spin electrons. The effects can be checked experimentally by studying Coulomb blockadelike oscillations either for chiral strongly correlated electron quantum wires with point contact potential backscatterer, or for quantum wire connected with point contacts to external voltages (leads connected with bulk samples).

This work has been supported in part by the Deutsche Forschungsgemeinschaft under Grant No. Fr 737/2-1.
${ }^{1}$ C. L. Kane and M. P. A. Fisher, Phys. Rev. B 46, 15233 (1992).

${ }^{2}$ S. Ghoshal and A. B. Zamolodchikov, Int. J. Mod. Phys. A 9, 3841 (1994); P. Fendley and H. Saleur, Nucl. Phys. B428, 681 (1994); P. Fendley, A. W. W. Ludwig, and H. Saleur, Phys. Rev. Lett. 74, 3005 (1995).

${ }^{3}$ A. M. Tsvelik, Phys. Rev. B 52, 4366 (1995); P. A. de Sa and A. M. Tsvelik, ibid. 52, 3067 (1995).

${ }^{4}$ R. Fazio, F. W. J. Hekking, and A. A. Odintsov, Phys. Rev. Lett. 74, 1843 (1995); P. Fröjdh and H. Johannesson, ibid. 75, 300 (1995); S. Eggert, H. Johannesson, and A. Mattsson, ibid. 76, 1505 (1996).

${ }^{5}$ V. E. Korepin, A. G. Izergin, and N. M. Bogoliubov, Quantum Inverse Scattering Method, Correlation Functions and Algebraic Bethe Ansatz (Cambridge University Press, Cambridge, 1993).

${ }^{6}$ H. Asakawa and M. Suzuki, J. Phys. A 29, 225 (1996).

${ }^{7}$ V. T. Petrashov, V. N. Antonov, P. Delsing, and T. Claeson, Phys. Rev. Lett. 74, 5268 (1995); S. Tarucha, T. Honda, and T. Saku, Solid State Commun. 94, 413 (1995); F. P. Milliken, C. P. Umbach, and R. A. Webb, ibid. 97, 309 (1996).
${ }^{8}$ A. Yacoby, M. Heiblum, D. Mahalu, and H. Shtrikman, Phys. Rev. Lett. 74, 4047 (1995); A. Yacoby, R. Schuster, and M. Heiblum, Phys. Rev. B 53, 9583 (1996).

${ }^{9}$ F. C. Alcaraz, M. N. Barber, M. T. Batchelor, R. J. Baxter, and G. R. W. Quispel, J. Phys. A 20, 6397 (1987).

${ }^{10}$ F. Woynarovich, J. Phys. A 22, 4243 (1989).

${ }^{11}$ H. Frahm and V. E. Korepin, Phys. Rev. B 43, 5653 (1991).

${ }^{12}$ J. L. Cardy and D. C. Lewellen, Phys. Lett. B 259, 274 (1991).

${ }^{13}$ A. A. Zvyagin and I. V. Krive, Fiz. Nizk. Temp. 21, 687 (1995) [Low Temp. Phys. 21, 533 (1995)].

${ }^{14}$ L. I. Glazman and R. I. Shekhter, J. Phys. Condens. Matter 1, 5811 (1989); D. Yue, L. I. Glazman, and K. A. Matveev, Phys. Rev. B 49, 1966 (1994).

${ }^{15}$ H. J. de Vega and F. Woynarowich, Nucl. Phys. B251, 439 (1985).

${ }^{16}$ F. Woynarovich, H.-P. Eckle, and T. T. Truong, J. Phys. A 22, 4027 (1989).

${ }^{17}$ A. A. Zvyagin and P. Schlottmann (unpublished).

${ }^{18}$ H. Frahm and A. A. Zvyagin (unpublished). 